\title{
Retinal mosaics: pattern formation driven by local interactions between homotypic neighbors
}

\author{
Benjamin E. Reese* \\ Neuroscience Research Institute, University of California at Santa Barbara, Santa Barbara, CA, USA \\ *Correspondence: breese@psych.ucsb.edu
}

Individual types of retinal neurons are distributed across the retina as regular arrays of cells, commonly referred to as "retinal mosaics" (Wässle and Riemann, 1978). This regularity in their patterning is generally assumed to ensure that all locations across the retina are sub-served by every type of retinal neuron, each participating in their own unique way to the local computations carried out before transmission of the visual signal to higher visual centers in the brain. The extent to which each retinal cell type evidences spatial order varies, with some mosaics being only slightly more regular than random distributions of cells (Reese, 2008a). With the exception of certain photoreceptor mosaics however (particularly in non-mammalian retinas), the spatial order present within the more regular of these mosaics lacks any higher-order lattice-like patterning (Cook, 2003), and simulations based on local-spacing rules that minimize proximity between immediate homotypic neighbors are sufficient to account for the spatial order present (Eglen, 2006). Indeed, the order present within a mosaic is generally independent of that in other mosaics (Rockhill et al., 2000), suggesting that the composition of retinal clones and the spatial relationships between them cannot account for the patterning in these mosaics. Rather, cells within a mosaic are free to move tangentially as they space themselves out, establishing territories surrounding each cell where the probability of a homotypic cell is lower than at further distances from the cell (Reese and Galli-Resta, 2002).

Functional retinal mosaics are commonly associated with the phenomenon of dendritic tiling, by which each cell in a mosaic extends its dendritic processes to "tessellate" the retinal surface by limiting further growth at the boundaries with homotypic dendritic fields, establishing a dendritic coverage of the retina of 1.0. Yet many regular retinal mosaics contain nerve cells with extensive dendritic overlap between their homotypic neighbors, for instance, the retinal horizontal cells and the starburst amacrine cells, having dendritic coverage factors 6-30 times greater, in the mouse retina (Reese, 2008b). Each of these cell types has been shown to disperse tangentially at a developmental stage presaging the emergence of the mature pattern (Galli-Resta et al., 1997; Raven et al., 2005), yet whether these cells move via active migration or by nuclear repositioning has been debated (Cook and Chalupa, 2000). Dendritic outgrowth at these stages is limited (Galli-Resta et al., 2002), but recent live imaging studies suggest these cell types may initially tessellate the retina with their dendrites before differentiating their mature, and vastly overlapping dendritic arbors (Huckfeldt et al., 2009). Ablating single horizontal cells at these early stages leads neighboring cells to extend their processes into the ablated territory, occasionally followed by somal repositioning nearer the center of the emerging dendritic field (Huckfeldt et al., 2009). These results suggest that homotypic interactions via developing dendritic arbors enable neighboring cells to space themselves apart, but the molecular means by which this interaction is achieved is unknown. A recent elegant study in the developing mouse retina reported in Nature now reveals two transmembrane proteins that participate in this homotypic recognition critical for intercellular repulsion in the mosaics of starburst amacrine cells and horizontal cells.

Kay et al. (2012) identified, via microarray analysis of purified retinal cell types harvested at P6, two genes expressed by only these cell types (Megf10 and Megf11; the former was also expressed in Müller glial cells). They confirmed selective expression in vivo, with Megflo preceding that of Megf11. Megf10 was detected prenatally in starburst amacrine cells as these nascent neuroblasts migrate into their mosaics in either the inner nuclear layer (INL) or ganglion cell layer (GCL).
Megf11, by contrast, was not detected until after birth in both starburst amacrine cells and horizontal cells. Both exhibited, via immunofluorescence, a distribution of labeling expected for membrane-associated proteins, outlining the soma, and dendritic processes. Indeed, each homolog encodes a transmembrane protein containing multiple EGF-like domains and a cytoplasmic domain with multiple binding sites for signal transduction, though until now, these proteins were implicated only as engulfment receptors for clearing degenerating cells. Kay and colleagues show, via gene knockout analysis, a conspicuous phenotype on the spatial ordering in these retinal mosaics without affecting the mosaics of other neighboring cell types.

The starburst amacrine cell mosaics in $M e g f 10^{-1-}$ retinas, but not $M e g f 11^{-1-}$ retinas, showed a striking loss of regularity, rendering them comparable to random simulations of cells matched in density and constrained by soma size. These mosaics lacked the minimal spacing constraint that generates their patterning. Curiously, the horizontal cell mosaics were only slightly

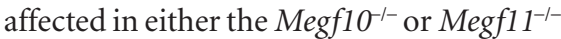
retinas, but were conspicuously impacted in the double-knockout, again being comparable to random distributions of cells. The double-knockout retina did not, however, impact the starburst amacrine cell mosaic any more than did knocking out Megf10 alone.

To investigate further the role of Megflo in mosaic formation, Kay et al. (2012) electroporated plasmid DNA into dividing cells of the newborn mouse retina, transfecting primarily later-generated rod photoreceptors, bipolar cells, Müller glial cells, and some amacrine cells (all of the starburst amacrine cells and horizontal cells are generated prenatally, and so escaped transfection). This protocol creates large fields of transfected retina in which the concentration of Megf10-positive processes is elevated (albeit misexpressed in 
other cell types), surrounded by regions free of transfection where the concentration is normal (being expressed only on the processes of horizontal cells and starburst amacrine cells). Regions within or outside large transfected patches were apparently normal with respect to their mosaic distribution. Of particular interest was the boundary-region, where starburst amacrine cells had vacated a rim of transfected territory, being misplaced outside of it, elevating the immediate density of starburst amacrine cells just outside the transfected region (and presumably reducing slightly the local density just beyond this elevated region). Using this same paradigm, the authors then showed that this same phenotype could be achieved when they transfected with a truncated form of Megf10, one lacking the cytoplasmic domain, indicating its role as a ligand driving this interaction. If however they transfected $\mathrm{Megf10^{-/ }}$ retinas, no misplacement of starburst amacrine cells was obtained, suggesting it must also be the receptor for this interaction.

Additional in vitro studies using transfected HEK293 cells showed that neighboring Megf10-positive cells form sharp if inter-digitated cellular borders, whereas if only one cell is positive, then neighboring cells overlap, extending fine filopodia upon one another. While such culture studies help to illustrate the dynamics of the interaction at the level of neighboring membranes, they raise as many questions about the mechanics of the interaction between cells in vivo, where the differentiation of neurons is so very different from this cell line in culture. The primary phenotype in this study is a histotypical one, a feature of the differentiated retina evidenced only by studying the population of cells in vivo. Cell number is not impacted, somal size and shape appear normal, and all other reported aspects of the retina appear to be unchanged. Indeed, the authors examined the dendritic morphology of single starburst amacrine (and horizontal) cells in these knockout (and double-knockout) retinas, suggesting their final differentiated appearance is unaffected.

Much remains, consequently, to be understood about how Megf-positive cells interact during development to control cellular positioning within the retina. For instance, do these cells engage in a brief period of contact-mediated inhibition of further dendritic outgrowth, suggested by the cell culture studies, during which somata center themselves via nuclear repositioning based on proximity to their neighbors (perhaps mediated by the length of those processes)? And if so, what then turns off this homotypic repulsion, permitting extensive dendritic outgrowth and overlap, given that gene expression is not down-regulated until much later during development? Furthermore, what is the origin of the pattern present in the mosaics of these mutant retinas? The authors report those mosaics to be spatially indiscriminable from random distributions of cells, yet their illustrations reveal what appear to be occasional clumps of cells suggestive of a clustering phenotype (Ding et al., 2009), raising the possibility that removing this homophilic repellent unmasks an attractive or adhesive interaction between like-type somata (Fuerst et al., 2009). Further analysis of the prevalence of such a clustering phenotype, and assessment of the dendritic arbors associated with such clustered cells, is warranted. Other studies show that these mosaics have some degree of spatial regularity before they undergo tangential dispersion (Raven et al., 2005), implying a limited spatial order already imposed by the periodic assignment of cellular fate (Tyler et al., 2005). That their total numbers in maturity are under tight genetic control would appear to be at odds with initial fate assignments conforming to complete spatial randomness (Whitney et al., 2011), suggesting some loss of that initial order in these mutant retinas, perhaps by the passive displacement of these cells subsequent to the genesis of later-produced cell types. Megf $10^{--}$retinas show a degradation of spatial order in the starburst amacrine cell mosaic at birth, when new cells are being added to the mosaic, suggesting that Megf10 (and Megf11) both maintain and improve this initial cellular spacing as later-born cell types migrate to their respective layers during subsequent retinal expansion.

\section{REFERENCES}

Cook, J. E. (2003). "Spatial regularity among retinal neurons," in The Visual Neurosciences, eds L. M. Chalupa and J. S. Werner (Cambridge: MIT Press), 463-477.

Cook, J. E., and Chalupa, L. M. (2000). Retinal mosaics: new insights into an old concept. Trends Neurosci. 23, 26-34

Ding, Q., Chen, H., Xie, X., Libby, R. T., Tian, N., and Gan, L. (2009). BARHL2 differentially regulates the development of retinal amacrine and ganglion neurons. J. Neurosci. 29, 3992-4003.
Eglen, S. J. (2006). Development of regular cellular spacing in the retina: theoretical models. Math. Med. Biol. 23, 79-99.

Fuerst, P. G., Bruce, F., Tian, M., Wei, W., Elstrott, J., Feller, M. B., Erskine, L., Singer, J. H., and Burgess, R. W. (2009). DSCAM and DSCAML1 function in self-avoidance in multiple cell types in the developing mouse retina. Neuron 64, 484-497.

Galli-Resta, L., Novelli, E., and Viegi, A. (2002). Dynamic microtubule-dependent interactions position homotypic neurons in regular monolayered arrays during retinal development. Development 129 , 3803-3814.

Galli-Resta, L., Resta, G., Tan, S.-S., and Reese, B. E. (1997). Mosaics of islet-1 expressing amacrine cells assembled by short range cellular interactions. J. Neurosci. 17, 7831-7838.

Huckfeldt, R. M., Schubert, T., Morgan, J. L., Godinho, L., Di Cristo, G., Huang, Z. J., and Wong, R. O. L. (2009). Transient neurites of retinal horizontal cells exhibit columnar tiling via homotypic interactions. Nat. Neurosci. 12, 35-43.

Kay, J. N., Chu, M. W., and Sanes, J. R. (2012). MEGF10 and MEGF11 mediate homotypic interactions required for mosaic spacing of retinal neurons. Nature 483, 465-469.

Raven, M. A., Stagg, S. B., Nassar, H., and Reese, B. E. (2005).Developmental improvement in the regularity and packing of mouse horizontal cells: implications for mechanisms underlying mosaic pattern formation. Vis. Neurosci. 22, 569-573.

Reese, B. E. (2008a). "Mosaics, tiling and coverage by retinal neurons," in The Senses: Vision, eds R. H. Masland and T. Albright (Oxford: Elsevier), 439-456.

Reese, B. E. (2008b). "Mosaic architecture of the mouse retina," in Eye, Retina, and Visual Systems of the Mouse, eds L. M. Chalupa and R. W. Williams (Cambridge: MIT Press), 147-155.

Reese, B. E., and Galli-Resta, L. (2002). The role of tangential dispersion in retinal mosaic formation. Prog. Retin. Eye Res. 21, 153-168.

Rockhill, R. L., Euler, T., and Masland, R. H. (2000). Spatial order within but not between types of retinal neurons. Proc. Natl. Acad. Sci. U.S.A. 97, 2303-2307.

Tyler, M. J., Carney, L. H., and Cameron, D. A. (2005). Control of cellular pattern formation in the vertebrate inner retina by homotypic regulation of cell-fate decisions. J. Neurosci. 25, 4565-4576.

Wässle, H., and Riemann, H. J. (1978). The mosaic of nerve cells in the mammalian retina. Proc. R. Soc. Lond. B Biol. Sci. 200, 441-461.

Whitney, I. E., Raven, M. A., Ciobanu, D. C., Poché, R. A., Ding, Q., Elshatory, Y., Gan, L., Williams, R. W., and Reese, B. E. (2011). Genetic modulation of horizontal cell number in the mouse retina. Proc. Natl. Acad. Sci. U.S.A. 108, 9697-9702.

Received:02 April 2012; accepted: 17 April 2012; published online: 04 May 2012.

Citation: Reese BE (2012) Retinal mosaics: pattern formation driven by local interactions between homotypic neighbors. Front. Neural Circuits 6:24. doi: 10.3389/ fncir.2012.00024

Copyright (C) 2012 Reese. This is an open-access article distributed under the terms of the Creative Commons Attribution Non Commercial License, which permits non-commercial use, distribution, and reproduction in other forums, provided the original authors and source are credited. 